\title{
A New Analytical Procedure to Solve Two Phase Flow in Tubes
}

\author{
Terry E. Moschandreou ${ }^{1,2}$ \\ 1 School of Applied Science and Technology, Fanshawe College, 1001 Fanshawe College Blvd, London, \\ ON N5Y 5R6, Canada; tmoschandreou@fanshawec.ca; Tel.: +1-519-452-4430 \\ 2 Department of Medical Biophysics, Schulich School of Medicine and Dentistry, Western University, \\ London, ON N6A 5C1, Canada; tmoschan@uwo.ca
}

Received: 8 April 2018; Accepted: 21 May 2018; Published: 23 May 2018

\begin{abstract}
A new formulation for a proposed solution to the 3D Navier-Stokes Equations in cylindrical co-ordinates coupled to the continuity and level set convection equation is presented in terms of an additive solution of the three principle directions in the radial, azimuthal and $z$ directions of flow and a connection between the level set function and composite velocity vector for the additive solution is shown. For the case of a vertical tube configuration with small inclination angle, results are obtained for the level set function defining the interface in both the radial and azimuthal directions. It is found that the curvature dependent part of the problem alone induces sinusoidal azimuthal interfacial waves whereas when the curvature together with the equation for the composite velocity is considered oscillating radial interfacial waves occur. The implications of two extremes indicate the importance of looking at the full equations including curvature.
\end{abstract}

Keywords: fluid dynamics; two phase flow; level set function; cylindrical coordinates; continuity equation

\section{Introduction}

Annular two phase flow is frequently experienced in industrial applications, for example, in power generation plants and heat removal devices. Applications include transfer lines in pipes (gas-liquid oil wells), evaporators and condensers. This flow regime involves a liquid phase flow in the form of a thin film along the pipe wall and a core region of a gas phase where there are droplets entrained within the gas. An important feature of annular pipe flows is the formation of waves at the core/film interface. Thus the Navier Stokes equations which describe the flow problem must be addressed inorder to determine if the 3-D velocity components exhibit wave-like characteristics. The Navier Stokes equations have been dealt with extensively in the literature for both analytical [1,2] and numerical solutions $[3,4]$. The level set method, has been used originally as a numerical technique for tracking interfaces and shapes [5,6] and has been increasingly applied to various areas of engineering and applied mathematics. In the level set method, contours or surfaces are represented as the zero level set of a higher dimensional function called a level set function. This can be the distance from the particular phase of material to the interface. For example in fracture mechanics level set methods have been used to track the shape around a crack in two and three dimensions that is propagating with a sharp kink [7]. In addition, various applications in image segmentation have been used with corresponding active curve evolution algorithms [8,9]. Reachability analysis is frequently used to study the safety of control systems. Using exact reachability operators for nonlinear hybrid systems is presented in [10]. An algorithm for determining reachable sets and synthesizing control laws is implemented using level set methods in [10]. Various models used to compute the interaction of 3D incompressible fluids with elastic membranes or bodies, rely on the use of level set functions [11], to capture the fluid-solid interfaces and to measure elastic stresses that have been used. In [11] the computation of equilibrium 
shapes of biological vesicles is presented and numerical simulations of spontaneous cardiomoyocyte contractions is presented. A conservative method of level set type for moving interfaces in divergence free velocity fields is presented in [12,13]. The method in [13] was coupled to a Navier-Stokes solver for incompressible two phase flow with surface tension. Wave phenomena is known to exist at the interface of two phase immiscible flows [14]. In the present paper, we present a level set method for moving interfaces for such velocity fields which are coupled to Navier-Stokes equations for two phase flows in tubes. The need for a cylindrical co-ordinate system is apparent as most of the industrial applications involve assemblies of round pipes and therefore a simple model using a straight tube is necessary to obtain insight into the more complex problem found there. The novelty of the present work is to reveal an analytical approach in solving the 3D cylindrical Navier-Stokes equations where the three principle directions of flow, in radial, azimuthal and longitudinal directions are summed to form a new composite vector velocity expression. In this light, we propose to solve a curvature only formulation of the governing equation for the level set function and one in which curvature is added to the governing equation in the large time evolution for the level set function and hence composite velocity formulation. The importance of these two extremes is to get some insight into the nature of and existence of interfacial waves and level set function for the interface and velocity profiles in annular flow. Also it is hoped that the method outlined in this work will lead to eventually solve the full problem analytically for the composite velocity with full curvature expression in cylindrical coordinates included. To the best of our findings this remains an open problem on the subject of two phase flows. Finally, the method is new in the sense that having an analytical solution allows us to solve for velocity and coupled temperature fields easier than employing numerical methods for certain cases. Our results for the limiting cases demonstrate how the velocity and level set function exhibits wave like phenomea in both the radial and azimuthal directions of flow. The problem is solved by assuming the level set function is a product of a decreasing exponential term with a function of radial and azimuthal coordinates. It is shown that when the rate of decrease of time dependent term is very high the level set function exhibits oscillations with higher frequency and lower amplitude and eventually approaches a hyperbolic tan function which is a steady state level set function. Further results showing composite velocity are also shown.

\subsection{Level Sets in Cylindrical Co-Ordinates}

Let $\phi$ be a level set function $[5,15]$. The gradient of the level set function in cylindrical co-ordinates is defined as:

$$
\nabla \phi=\left(\frac{\partial \phi}{\partial r}, \frac{1}{r} \frac{\partial \phi}{\partial \theta}, \frac{\partial \phi}{\partial z}\right)
$$

The mean curvature, $\kappa$, of the interface defined by the zero isocontour of the level set function $\phi[5,15]$, is the divergence of the normal to the interface given by

$$
\vec{n}=\frac{\nabla \phi}{|\nabla \phi|}
$$

Thus it can be expressed as:

$$
\kappa=-\nabla \cdot \vec{n}
$$


The mean curvature $\kappa$ of a dynamic surface $\phi(r, \theta, z, t)=\psi(r, \theta, t)+z$ in cylindrical co-ordinates is,

$$
\begin{array}{r}
\kappa=\frac{1}{S}\left[( \frac { \partial } { \partial r } \phi ( r , \theta , z , t ) ) \left(r^{2}\left(\left(\frac{\partial}{\partial r} \phi(r, \theta, z, t)\right)^{2}+1\right)+\right.\right. \\
\left.2\left(\frac{\partial}{\partial \theta} \phi(r, \theta, z, t)\right)\left(\frac{\partial}{\partial \theta} \phi(r, \theta, z, t)-r \frac{\partial^{2}}{\partial \theta \partial r} \phi(r, \theta, z, t)\right)\right)+ \\
r\left(\left(\frac{\partial}{\partial \theta} \phi(r, \theta, z, t)\right)^{2}+r^{2}\right) \frac{\partial^{2}}{\partial r^{2}} \phi(r, \theta, z, t)+ \\
\left.r\left(\frac{\partial^{2}}{\partial \theta^{2}} \phi(r, \theta, z, t)\right)\left(\left(\frac{\partial}{\partial r} \phi(r, \theta, z, t)\right)^{2}+1\right)\right]
\end{array}
$$

where

$$
S=\left(2 r^{2}\left(\left(\frac{\partial}{\partial r} \phi(r, \theta, z, t)\right)^{2}+1\right)+2\left(\frac{\partial}{\partial \theta} \phi(r, \theta, z, t)\right)^{2}\right)^{3 / 2}
$$

The geometric trace of a dynamic closed surface that bounds an open set can be represented implicitly as

$$
S=\{(r, \theta, z, t) \mid \phi(r, \theta, z, t)=0\}
$$

and for two phase flow has two separate regions where $\phi>0$ and $\phi<0$ respectively [15]. The surface evolution is determined by:

$$
\phi_{t}=\mathbf{u} \cdot \nabla \phi
$$

In this work the function $\phi$ is what is called a filter which has an effective width and will be defined on a small region near the interface. The form of the Navier-Stokes Equations that are not coupled to the interface of the two phases are:

$$
\frac{\partial \mathbf{u}}{\partial t}+(\mathbf{u} \cdot \nabla) \mathbf{u}=-\frac{1}{\rho} \nabla p+\mu \nabla^{2} \mathbf{u}+\frac{1}{\rho} \mathbf{F}
$$

where for cylindrical $(r, \theta, z)$ coordinate system, Laplace operator has the form

$$
\nabla^{2}=(\underbrace{\frac{\partial^{2}}{\partial r^{2}}+\frac{1}{r} \frac{\partial}{\partial r}}_{\frac{1}{r} \frac{\partial}{\partial r}\left(r \frac{\partial}{\partial r}\right)}+\frac{1}{r^{2}} \frac{\partial^{2}}{\partial \theta^{2}}+\frac{\partial^{2}}{\partial z^{2}})
$$

the gradient is given by Equation (1) and $\mathbf{u}$ is the three dimensional velocity of the flow field and is described further below.

\subsection{A New Composite Velocity Formulation}

The 3D cylindrical incompressible unsteady Navier-Stokes equations coupled to interface convection equation are written in expanded form, for each component, $u_{r}, u_{\theta}$ and $u_{z}$, where the remaining non linear terms appearing in full Navier-Stokes equations are suppressed for the time being:

$$
\begin{gathered}
\frac{\partial u_{r}}{\partial t}+u_{r} \frac{\partial u_{r}}{\partial r}+\frac{u_{\theta}}{r} \frac{\partial u_{r}}{\partial \theta}+u_{z} \frac{\partial u_{r}}{\partial z}-\frac{\mu}{\rho}\left(-\frac{u_{r}}{r^{2}}+\frac{\partial^{2} u_{r}}{\partial r^{2}}+\frac{1}{r} \frac{\partial u_{r}}{\partial r}+\frac{1}{r^{2}} \frac{\partial^{2} u_{r}}{\partial \theta^{2}}+\frac{\partial^{2} u_{r}}{\partial z^{2}}\right)+ \\
\frac{1}{\rho} \frac{\partial p}{\partial r}-F g_{r}-\frac{1}{\rho} \sigma \kappa \frac{\partial \phi}{\partial r}=0
\end{gathered}
$$




$$
\begin{gathered}
\frac{\partial u_{\theta}}{\partial t}+u_{r} \frac{\partial u_{\theta}}{\partial r}+\frac{u_{\theta}}{r} \frac{\partial u_{\theta}}{\partial \theta}+u_{z} \frac{\partial u_{\theta}}{\partial z}-\frac{\mu}{\rho}\left(-\frac{u_{\theta}}{r^{2}}+\frac{\partial^{2} u_{\theta}}{\partial r^{2}}+\frac{1}{r} \frac{\partial u_{\theta}}{\partial r}+\frac{1}{r^{2}} \frac{\partial^{2} u_{\theta}}{\partial \theta^{2}}+\frac{\partial^{2} u_{\theta}}{\partial z^{2}}\right)+ \\
\frac{1}{\rho} \frac{\partial p}{\partial \theta}-F g_{\theta}-\frac{1}{\rho} \sigma \kappa \frac{1}{r} \frac{\partial \phi}{\partial \theta}=0 \\
\frac{\partial u_{z}}{\partial t}+u_{r} \frac{\partial u_{z}}{\partial r}+\frac{u_{\theta}}{r} \frac{\partial u_{z}}{\partial \theta}+u_{z} \frac{\partial u_{z}}{\partial z}-\frac{\mu}{\rho}\left(\frac{\partial^{2} u_{z}}{\partial r^{2}}+\frac{1}{r} \frac{\partial u_{z}}{\partial r}+\frac{1}{r^{2}} \frac{\partial^{2} u_{z}}{\partial \theta^{2}}+\frac{\partial^{2} u_{z}}{\partial z^{2}}\right)+ \\
\frac{1}{\rho} \frac{\partial p}{\partial z}-F g_{z}-\frac{1}{\rho} \sigma \kappa \frac{\partial \phi}{\partial z}=0
\end{gathered}
$$

and where $u_{r}$ is the radial component of velocity, $u_{\theta}$ is the azimuthal component and $u_{z}$ is the component along the pipe in the direction of fully developed upward flow, $\rho$ is density, $\mu$ is dynamic viscosity, $F g_{r}, F g_{\theta}, F g_{z}$ are body forces on fluid and $\sigma$ is surface interfacial tension. Introducing the following definition,

$$
\overrightarrow{\vec{L}}=\frac{\partial \phi}{\partial r} \vec{e}_{r}+\frac{1}{r} \frac{\partial \phi}{\partial \theta} \overrightarrow{e_{\theta}}+\frac{\partial \phi}{\partial z} \vec{k}
$$

Multiplying Equations (8)-(10) by unit vectors $\overrightarrow{e_{r}}, \overrightarrow{e_{\theta}}$ and $\vec{k}$ respectively and adding Equations (8)-(10) gives the following equation, for $\vec{L}=u_{r} \overrightarrow{e_{r}}+u_{\theta} \overrightarrow{e_{\theta}}+u_{z} \vec{k}$ and $\overrightarrow{\vec{L}}$

$$
\frac{\partial \vec{L}}{\partial t}+u_{r} \frac{\partial \vec{L}}{\partial r}+\frac{u_{\theta}}{r} \frac{\partial \vec{L}}{\partial \theta}+u_{z} \frac{\partial \vec{L}}{\partial z}-\frac{\mu}{\rho}\left(-\frac{\vec{L}}{r^{2}}+\frac{\partial^{2} \vec{L}}{\partial r^{2}}+\frac{1}{r} \frac{\partial \vec{L}}{\partial r}+\frac{1}{r^{2}} \frac{\partial^{2} \vec{L}}{\partial \theta^{2}}+\frac{\partial^{2} \vec{L}}{\partial z^{2}}\right)+\frac{1}{\rho} P_{T}-F_{T}-\frac{1}{\rho} \sigma \kappa \overline{\vec{L}}=0
$$

The level set function $\phi$ is governed by Equation (7), which when expanded becomes in cylindrical co-ordinates:

$$
\phi_{t}+u_{r} \frac{\partial \phi}{\partial r}+\frac{u_{\theta}}{r} \frac{\partial \phi}{\partial \theta}+u_{z} \frac{\partial \phi}{\partial z}=0
$$

The continuity equation in cylindrical co-ordinates is

$$
\frac{\partial \rho}{\partial t}+u_{r} \frac{\partial \rho}{\partial r}+\frac{u_{\theta}}{r} \frac{\partial \rho}{\partial \theta}+u_{z} \frac{\partial \rho}{\partial z}=-\rho\left(\frac{\partial u_{r}}{\partial r}+\frac{1}{r} \frac{\partial u_{\theta}}{\partial \theta}+\frac{\partial u_{z}}{\partial z}\right)
$$

Multiply Equation (11) by $\frac{\rho}{\mu} \phi$ :

$$
\begin{gathered}
\frac{\rho}{\mu}\left[\phi \frac{\partial \vec{L}}{\partial t}+\phi u_{r} \frac{\partial \vec{L}}{\partial r}+\phi \frac{u_{\theta}}{r} \frac{\partial \vec{L}}{\partial \theta}+\phi u_{z} \frac{\partial \vec{L}}{\partial z}-\right. \\
\left.\frac{\mu}{\rho} \phi\left(-\frac{\vec{L}}{r^{2}}+\frac{\partial^{2} \vec{L}}{\partial r^{2}}+\frac{1}{r} \frac{\partial \vec{L}}{\partial r}+\frac{1}{r^{2}} \frac{\partial^{2} \vec{L}}{\partial \theta^{2}}+\frac{\partial^{2} \vec{L}}{\partial z^{2}}\right)+\frac{1}{\rho} \phi P_{T}-\phi F_{T}-\frac{1}{\rho} \sigma \phi \kappa \overrightarrow{\vec{L}}\right]=0
\end{gathered}
$$

Multiply Level set function convection Equation (12) by $\frac{\rho}{\mu} \vec{L}$ :

$$
\frac{\rho}{\mu}\left[\vec{L} \frac{\partial \phi}{\partial t}+\vec{L} u_{r} \frac{\partial \phi}{\partial r}+\vec{L} \frac{u_{\theta}}{r} \frac{\partial \phi}{\partial \theta}+\vec{L} u_{z} \frac{\partial \phi}{\partial z}\right]=0
$$

Adding Equations (14) and (15) gives

$$
\begin{gathered}
\frac{\rho}{\mu}\left[\phi \frac{\partial \vec{L}}{\partial t}+\vec{L} \frac{\partial \phi}{\partial t}+\phi u_{r} \frac{\partial \vec{L}}{\partial r}+\vec{L} u_{r} \frac{\partial \phi}{\partial r}+\phi \frac{u_{\theta}}{r} \frac{\partial \vec{L}}{\partial \theta}+\vec{L} \frac{u_{\theta}}{r} \frac{\partial \phi}{\partial \theta}+\phi u_{z} \frac{\partial \vec{L}}{\partial z}+\vec{L} u_{z} \frac{\partial \phi}{\partial z}-\right. \\
\left.\frac{\mu}{\rho} \phi\left(-\frac{\vec{L}}{r^{2}}+\frac{\partial^{2} \vec{L}}{\partial r^{2}}+\frac{1}{r} \frac{\partial \vec{L}}{\partial r}+\frac{1}{r^{2}} \frac{\partial^{2} \vec{L}}{\partial \theta^{2}}+\frac{\partial^{2} \vec{L}}{\partial z^{2}}\right)+\frac{1}{\rho} \phi P_{T}-\phi F_{T}-\frac{1}{\rho} \sigma \phi \kappa \overrightarrow{\vec{L}}\right]=0
\end{gathered}
$$


By product rule we rewrite the previous equation as:

$$
\begin{gathered}
\frac{\rho}{\mu}\left[\frac{\partial(\phi \vec{L})}{\partial t}+\frac{\partial\left(\phi u_{r} \vec{L}\right)}{\partial r}+\frac{1}{r} \frac{\partial\left(\phi u_{\theta} \vec{L}\right)}{\partial \theta}+\frac{\partial\left(\phi u_{z} \vec{L}\right)}{\partial z}\right]- \\
\phi\left(-\frac{\vec{L}}{r^{2}}+\frac{\partial^{2} \vec{L}}{\partial r^{2}}+\frac{1}{r} \frac{\partial \vec{L}}{\partial r}+\frac{1}{r^{2}} \frac{\partial^{2} \vec{L}}{\partial \theta^{2}}+\frac{\partial^{2} \vec{L}}{\partial z^{2}}\right)+\frac{1}{\mu} \phi P_{T}-\frac{\rho}{\mu} \phi F_{T}-\frac{1}{\mu} \sigma \phi \kappa \overline{\vec{L}}=0
\end{gathered}
$$

It is noted that since $\vec{L}$ is a composite velocity term it must have units of length per time, and since $\phi$ is usually taken to be a distance function from the interface we can consider the following expression in terms of $\phi$ :

$$
\vec{L}=\frac{1}{\phi} \frac{\mu}{\rho} \overrightarrow{\vec{L}}
$$

where $\frac{\mu}{\rho}$ has SI units of $\mathrm{m}^{2} / \mathrm{s}$ and $\overline{\vec{L}}$ is defined previously and is of dimension $1 / \mathrm{cm}$ and $\phi$ is dimensionless, (See Appendix A for decomposition of $\frac{\mu}{\rho}$ ) where $\rho_{1}, \mu_{1}$ are density and dynamic viscosity of fluid 1 and $\rho_{2}, \mu_{2}$ are density and dynamic viscosity of fluid 2 separated by interface and we write

$$
\rho=\rho_{1}+\left(\rho_{2}-\rho_{1}\right) \phi, \mu=\mu_{1}+\left(\mu_{2}-\mu_{1}\right) \phi
$$

It is assumed that the density and viscosity of fluid 1 is negligible compared to fluid 2 . From Level set Equation (7),

$$
\vec{L}(\vec{L} \cdot \overrightarrow{\vec{L}})=-\vec{L}\left(\phi_{t}\right)
$$

From the first line of Equation (17) using Equation (18) we have a derivative term in $t$, first we write:

$$
\frac{\mu}{\rho} \overline{\vec{L}}=\frac{\left(\mu_{1}+\left(\mu_{2}-\mu_{1}\right) \phi(r, \theta, z, t)\right) \overline{\vec{L}}(r, \theta, z, t)}{\rho_{1}+\left(\rho_{2}-\rho_{1}\right) \phi(r, \theta, z, t)}
$$

and for $\rho_{1}$ relatively small in comparison to $\rho_{2}$ and for $\mu_{1}$ relatively small to $\mu_{2}$ we obtain

$$
\frac{\partial(\phi \vec{L})}{\partial t}=\frac{\left(\frac{\partial}{\partial t} \overrightarrow{\vec{L}}(r, \theta, z, t)\right) \mu_{2}}{\rho_{2}}
$$

and for derivative terms in $r, \theta, z$, for $\rho_{1}$ small relative to $\rho_{2}$ and for $\mu_{1}$ small relative to $\mu_{2}$ from the first line of Equation (17) it can be proven that,

$$
\begin{gathered}
\frac{\partial\left(\phi u_{r} \vec{L}\right)}{\partial r}+\frac{1}{r} \frac{\partial\left(\phi u_{\theta} \vec{L}\right)}{\partial \theta}+\frac{\partial\left(\phi u_{z} \vec{L}\right)}{\partial z}= \\
\frac{\mu_{2}}{\rho_{2}}\left[\left(\frac{\partial}{\partial r} u_{r}(r, \theta, z, t)\right) \overline{\vec{L}}(r, \theta, z, t)+u_{r}(r, \theta, z, t) \frac{\partial}{\partial r} \overrightarrow{\vec{L}}(r, \theta, z, t)+\frac{u_{\theta}}{r}(r, \theta, z, t) \frac{\partial}{\partial \theta} \overline{\vec{L}}(r, \theta, z, t)+\right. \\
\left.\frac{1}{r}\left(\frac{\partial}{\partial \theta} u_{\theta}(r, \theta, z, t)\right) \overline{\vec{L}}(r, \theta, z, t)+u_{z}(r, \theta, z, t) \frac{\partial}{\partial z} \overrightarrow{\vec{L}}(r, \theta, z, t)+\overline{\vec{L}}(r, \theta, z, t) \frac{\partial}{\partial z} u_{z}(r, \theta, z, t)\right]
\end{gathered}
$$

\subsection{Special Case Solution}

In this section, it is assumed that the tube is in a vertical configuration with a small inclination angle, with assumption that $\rho_{2}$ and $\mu_{2}$ are nonzero terms for fluid 2 whereas fluid 1 has approximately zero density and viscosity. This is an idealization of the full problem which is amenable only to 
numerical treatment. It is worthy to notice that part of Equation (22) can be rewritten using the time dependent continuity equation Equation (13),

$$
\begin{gathered}
-\rho \times\left(\overrightarrow{\vec{L}} \frac{\partial}{\partial r} u_{r}(r, \theta, z, t)+\frac{\overrightarrow{\vec{L}} \frac{\partial}{\partial \theta} u_{\theta}(r, \theta, z, t)}{r}+\overrightarrow{\bar{L}} \frac{\partial}{\partial z} u_{z}(r, \theta, z, t)\right)=\overline{\vec{L}}\left(\frac{\partial \rho}{\partial t}+\vec{L} \cdot \nabla \rho\right) \\
\nabla \rho=\rho_{2} \nabla \phi
\end{gathered}
$$

Use of Equation (17), Equations (18)-(24) gives a non-linear PDE

$$
\begin{gathered}
\frac{\rho}{\mu} \frac{\partial(\phi \vec{L})}{\partial t}+\frac{\rho}{\mu}\left(\vec{L} \cdot \nabla \overrightarrow{\vec{L}}+\frac{1}{\rho} \overrightarrow{\vec{L}} \frac{\partial \rho}{\partial t}\right)+\frac{1}{\rho} \frac{\rho \rho_{2}}{\mu} \overrightarrow{\vec{L}} \vec{L} \cdot \nabla \phi- \\
\phi\left(-\frac{\vec{L}}{r^{2}}+\frac{\partial^{2} \vec{L}}{\partial r^{2}}+\frac{1}{r} \frac{\partial \vec{L}}{\partial r}+\frac{1}{r^{2}} \frac{\partial^{2} \vec{L}}{\partial \theta^{2}}+\frac{\partial^{2} \vec{L}}{\partial z^{2}}\right)+\frac{1}{\mu} \phi \overrightarrow{P_{T}}-\frac{\rho}{\mu} \phi \overrightarrow{F_{T}}-\frac{1}{\mu} \sigma \phi \kappa \overrightarrow{\vec{L}}=0
\end{gathered}
$$

Using Equation (4) and incorporating the curvature $\kappa$, and using Equation (18) and dot product in Equation (19), with the assumption of small inclination angle, Equation (25) for fully developed flow in the vertical $z$ direction becomes,

$$
\begin{gathered}
\frac{\mu_{2} \frac{\partial}{\partial t} \overrightarrow{\vec{L}}(r, \theta, t)}{\rho_{2} \phi}+\frac{\rho}{\mu} \vec{L} \cdot \nabla \overline{\vec{L}}-\frac{\vec{L}(r, \theta, t)}{r^{2}}+\frac{\partial^{2}}{\partial r^{2}} \vec{L}(r, \theta, t)+\frac{\frac{\partial}{\partial r} \vec{L}(r, \theta, t)}{r}+\frac{\frac{\partial^{2}}{\partial \theta^{2}}(r, \theta, t)}{r^{2}}- \\
F z-\frac{\sigma}{2 \mu_{2} \sqrt{2} \phi} \frac{\left(\frac{\partial}{\partial r} \phi(r, \theta, t)\right)^{2}}{r} \frac{1}{\sqrt{-\frac{\partial \partial}{\phi(t, \theta, t)}}}=0
\end{gathered}
$$

$F z$ is force of gravity in inclined tube. Incorporating Equation (18) and partial fraction decomposition for $\frac{\mu}{\rho}$ found in Appendix A, Equation(26) becomes for $\rho_{1}$ and $\mu_{1}$ negligibly small in comparison to $\rho_{2}$ and $\mu_{2}$ respectively of fluid 2 ,

$$
\begin{gathered}
2 \frac{\left(\frac{\partial}{\partial r} F(r, \theta)\right)^{3} \mu_{2}}{\rho_{2}(F(r, \theta))^{3}}+\frac{\left(\frac{\partial^{3}}{\partial r^{3}} F(r, \theta)\right) \mu 2}{\rho_{2} F(r, \theta)}-\frac{\sigma}{2 \mu_{2} \sqrt{2}} \frac{\left(\frac{\partial}{\partial r} F(r, \theta)\right)^{2}}{\sqrt{-\alpha} e^{-\alpha t} F(r, \theta)}-F z-\frac{\alpha \mu_{2} \frac{\partial}{\partial r} F(r, \theta)}{\rho_{2} F(r, \theta)}+ \\
2 \frac{\left(\frac{\partial}{\partial r} F(r, \theta)\right)\left(\frac{\partial}{\partial \theta} F(r, \theta)\right)^{2} \mu_{2}}{\rho_{2}(F(r, \theta))^{3} r^{2}}-2 \frac{\left(\frac{\partial}{\partial \theta} F(r, \theta)\right)\left(\frac{\partial^{2}}{\partial \theta \partial r} F(r, \theta)\right) \mu_{2}}{\rho 2(F(r, \theta))^{2} r^{2}}- \\
\frac{\left(\frac{\partial}{\partial r} F(r, \theta)\right)\left(\frac{\partial^{2}}{\partial \theta^{2}} F(r, \theta)\right) \mu_{2}}{\rho_{2}(F(r, \theta))^{2} r^{2}}+\frac{\left(\frac{\partial^{3}}{\partial \theta^{2} \partial r} F(r, \theta)\right) \mu_{2}}{\rho_{2} F(r, \theta) r^{2}}-\frac{\left(\frac{\partial}{\partial r} F(r, \theta)\right) \mu_{2}}{\rho_{2} F(r, \theta) r^{2}}+ \\
\frac{\left(\frac{\partial^{2}}{\partial r^{2}} F(r, \theta)\right) \mu_{2}}{\rho_{2} F(r, \theta) r}-\frac{\left(\frac{\partial}{\partial r} F(r, \theta)\right)^{2} \mu_{2}}{\rho_{2}(F(r, \theta))^{2} r}-3 \frac{\left(\frac{\partial^{2}}{\partial r^{2}} F(r, \theta)\right)\left(\frac{\partial}{\partial r} F(r, \theta)\right) \mu_{2}}{\rho_{2}(F(r, \theta))^{2}}=0
\end{gathered}
$$

where we have solved Equation (26) using the assumption of small inclination of tube, and have subsitituted $\overline{\vec{L}}=\nabla \phi$ and then set $\phi=e^{\alpha t} F(r, \theta)$ for $\alpha<0$ in Equation(26). This leads to Equation (27). 
It can be proven that $F(r, \theta)$ is multiplicatively separable when term in $t$ is dropped or $t$ gets large since exponential term is in the denominator for $\alpha<0$, thus, $F(r, \theta)=f(r) g(\theta)$ and we obtain the following,

$$
\begin{gathered}
\frac{\mathrm{d}^{3}}{\mathrm{~d} r^{3}} f(r)=\frac{f(r) F z \rho_{2}}{\mu_{2}}+\alpha \frac{\mathrm{d}}{\mathrm{d} r} f(r)+3 \frac{\left(\frac{\mathrm{d}^{2}}{\mathrm{~d} r^{2}} f(r)\right) \frac{\mathrm{d}}{\mathrm{d} r} f(r)}{f(r)}-2 \frac{\left(\frac{\mathrm{d}}{\mathrm{d} r} f(r)\right)^{3}}{(f(r))^{2}}- \\
\frac{\frac{\mathrm{d}^{2}}{\mathrm{~d} r^{2}} f(r)}{r}+\frac{\left(\frac{\mathrm{d}}{\mathrm{d} r} f(r)\right)^{2}}{f(r) r}+\frac{\frac{\mathrm{d}}{\mathrm{d} r} f(r)}{r^{2}}
\end{gathered}
$$

It can be shown that $g(\theta)$ is an arbitrary function of $\theta$, for which one chooses $g(\theta)$ to be constant and large inorder to be consistent with the omission of the term in $e^{\alpha t}$ in Equation (27).

Consideration of Curvature Alone

Secondly we solve the curvature pde given in Equation (4). For small inclination angle of tube Equation (4) reduces to,

$$
\begin{gathered}
-\left(\frac{\partial}{\partial r} \phi(r, \theta, t)\right) r^{2}\left(\frac{\partial}{\partial t} \phi(r, \theta, t)\right) \phi(r, \theta, t)+r^{3} \frac{\partial^{2}}{\partial r^{2}} \phi(r, \theta, t)- \\
r\left(\frac{\partial^{2}}{\partial \theta^{2}} \phi(r, \theta, t)\right)\left(\frac{\partial}{\partial t} \phi(r, \theta, t)\right) \phi(r, \theta, t)=0
\end{gathered}
$$

which is separable into,

$$
\begin{aligned}
\frac{\mathrm{d}}{\mathrm{d} r} f 1(r) & =\frac{c_{1} f 1(r)}{r} \\
\frac{\mathrm{d}^{2}}{\mathrm{~d} \theta^{2}} f 2(\theta) & =c_{2} f 2(\theta) \\
\frac{\mathrm{d}}{\mathrm{d} t} f 3(t) & =\frac{c_{3}}{f 3(t)}
\end{aligned}
$$

where $c_{3}$ is sufficiently large. For constant $c_{2}$ negative there is a sinusoidal component of the azimuthal part of $\phi(r, \theta, t)=f 1(r) f 2(\theta) f 3(t)$.

\section{Results and Discussion}

An analytical solution is found to exist for Equation (28) and is given by the following

$$
f(r)=\kappa_{1} \kappa_{2} \kappa_{3} \kappa_{4}^{-1}
$$

where

$$
\begin{aligned}
& \kappa_{1}=\mathrm{e}^{-1 / 2 \frac{F z J_{1}(\sqrt{-\alpha} r) \pi \mathbf{H}_{0}(\sqrt{-\alpha} r) \sqrt{-\alpha} \rho_{2} Y_{0}(\sqrt{-\alpha} r)}{\alpha^{2} \mu_{2}\left(J_{1}(\sqrt{-\alpha} r) Y_{0}(\sqrt{-\alpha} r)-J_{0}(\sqrt{-\alpha} r) Y_{1}(\sqrt{-\alpha} r)\right)}} \mathrm{e}^{1 / 2 \frac{F z Y_{1}(\sqrt{-\alpha} r) \pi \mathbf{H}_{0}(\sqrt{-\alpha} r) \sqrt{-\alpha} J_{0}(\sqrt{-\alpha} r) \rho_{2}}{\alpha^{2} \mu_{2}\left(J_{1}(\sqrt{-\alpha} r) Y_{0}(\sqrt{-\alpha} r)-J_{0}(\sqrt{-\alpha} r) Y_{1}(\sqrt{-\alpha} r)\right)}} \\
& \kappa_{2}=\mathrm{e}^{\frac{r F z \gamma_{1}(\sqrt{-\alpha}) J_{0}(\sqrt{-\alpha} r) \rho_{2}}{\alpha \mu_{2}\left(J_{1}(\sqrt{-\alpha} r) Y_{0}(\sqrt{-\alpha r})-J_{0}(\sqrt{-\alpha r}) Y_{1}(\sqrt{-\alpha} r)\right)}} \mathrm{e}^{\frac{C_{1} J_{1}(\sqrt{-\alpha} r) Y_{0}(\sqrt{-\alpha} r)}{\alpha\left(J_{1}(\sqrt{-\alpha}) Y_{0}(\sqrt{-\alpha} r)-J_{0}(\sqrt{-\alpha}) Y_{1}(\sqrt{-\alpha} r)\right)}} \\
& \kappa_{3}=\mathrm{e}^{\frac{J_{0}(\sqrt{-\alpha}) C_{3}}{\alpha r\left(J_{1}(\sqrt{-\alpha} r) Y_{0}(\sqrt{-\alpha} r)-J_{0}(\sqrt{-\alpha} r) Y_{1}(\sqrt{-\alpha} r)\right)}}
\end{aligned}
$$




$$
\begin{gathered}
\kappa_{4}=\mathrm{e}^{\frac{r F z \rho_{2} J_{1}(\sqrt{-\alpha} r) Y_{0}(\sqrt{-\alpha} r)}{\alpha \mu_{2}\left(J_{1}(\sqrt{-\alpha} r) Y_{0}(\sqrt{-\alpha} r)-J_{0}(\sqrt{-\alpha} r) Y_{1}(\sqrt{-\alpha} r)\right)}} \mathrm{e}^{\frac{J_{0}(\sqrt{-\alpha} r) Y_{1}(\sqrt{-\alpha} r) C_{1}}{\alpha\left(J_{1}(\sqrt{-\alpha} r) Y_{0}(\sqrt{-\alpha} r)-J_{0}(\sqrt{-\alpha}) Y_{1}(\sqrt{-\alpha} r)\right)}} \times \\
\mathrm{e}^{\frac{C_{2} Y_{0}(\sqrt{-\alpha} r)}{\alpha r\left(J_{1}(\sqrt{-\alpha} r) Y_{0}(\sqrt{-\alpha} r)-J_{0}(\sqrt{-\alpha} r) Y_{1}(\sqrt{-\alpha} r)\right)}}
\end{gathered}
$$

where $J_{0}(x), J_{1}(x)$ are Bessel functions of the first kind , $Y_{0}(x), Y_{1}(x)$ are Bessel functions of the second kind and $H_{0}(x)$ is a Struve function. $C_{1}, C_{2}$ and $C_{3}$ are constants taken to by each equal to unity. It is evident that the solution given by Equation (33) with $\kappa_{1}$ through $\kappa_{4}$ as in Equations (34)-(37) is positive everywhere with the exception at $r=0$ of being exactly zero. The convergence to zero is quite slow as $r \rightarrow 0^{+}$. The dynamic viscosity $\mu_{2}$ of fluid 2 which we take to be for water as a test case is $8.9 \times 10^{-3}$ dyn. s $/ \mathrm{cm}^{2}$ at a temperature of 25 degrees Celsius and at the same temperature the density $\rho_{2}$ of liquid water is $9.97044 \times 10^{-1} \mathrm{gm} / \mathrm{cm}^{3}$. Next we transform the center of the tube $r=0$ to the interface as in Figure 1. Mapping $r$ into $r-R_{i}$ in Equation (33), where $R_{i}$ is the radial value at the gas/water interface, it can be shown that $f(r)=0$ at $r=R_{i}$. We choose $R_{i}=0.85$, and let $r$ run to $r=1$. Results for the transformed function $f(r)$ are shown in Figures 2-6. In Figures 7 and 8 results are shown for composite velocity $L(r)$ which is $\vec{L}$ in the $r$ direction, i.e., $u_{r}$, see Figure 1 . Finally we approach a hyperbolic tan function as $\alpha$ becomes very large and negative for $f(r)$. This is due to the fact that $f(r)$ can be analytically continued to the interval closest to and to the left of $R_{i}$. It is evident that if we set $d=r-R_{i}$ to be the standard distance function from the interface [5,6] we can solve numerically for $d$ in Equations(33)-(37) and verify that $d$ is oscillating hence implying that the interface is oscillating back and forth in the radial direction. In addition I have compared to [16] for verification of a tan hyperbolic representation near the interface which is used there in a numerical approach. The solution given by Equation (33) can also be written in the form $1-\frac{\sin (\beta d)}{\beta d}$, where $\beta$ is chosen to be large and negative (not equal to $\alpha$ ). It was verified that $\phi$ could be shifted by scalar addition to avoid a velocity $\vec{L}$ that is undefined at zero as in Equation (18). The governing equations were extended by multiplying by the result of $\phi$ added to a nonzero constant. This results in a finite integral for $\vec{L}$ over the tube radius and meaningful comparisons to velocity measurements can be made as found in the literature [17], we have,

$$
u_{r}=-\frac{\mu_{2}}{\rho_{2}} \frac{\frac{\partial}{\partial r} \phi_{l}(r, \theta, t)}{\phi_{l}(r, \theta, t)}=-\frac{\mu_{2}}{\rho_{2}}\left(-\frac{\cos (r \beta)}{r}+\frac{\sin (r \beta)}{r^{2} \beta}\right)\left(A+1-\frac{\sin (r \beta)}{r \beta}\right)^{-1}
$$

where

$$
\phi_{l}=A+1-\frac{\sin (r \beta)}{r \beta}=A+\phi
$$

and $\phi_{l}$ is lifting of $\phi$ by a constant $A$. I have used data presented in [17] for $\mu_{2}, \rho_{2}, \frac{\mu_{2}}{\rho_{2}}=0.002 \mathrm{~m}^{2} / \mathrm{s}$ and in Equation (39), $A+1=-0.002689328$. Comparing Equation (33), to Equation (39) for $r=1 / 2 \mathrm{~m} \mathrm{[17],}$ $F z=0.3, \alpha=-1 \times 10^{6}$, constants $C_{1}, C_{2}$ and $C_{3}$ equal to unity and $\mu_{2}$ and $\rho_{2}$ as in [17], a solution for $\beta$ is obtained.

In Section 2.3 of [17] a grid convergence with various mesh resolutions were conducted for two phase flow simulation in an upward inclined two dimensional conducting pipe with Poiseuille flow at the inlet. Results in Figure 2 of [17] show the time histories of the velocity components, $u$ and $v$ in the $L_{2}$ norm. I have calculated the sum of Equation (38) from $r=d=0$ to $0.1\left(d=r-R_{i}=1 / 2-0.4=0.1\right)$, where it is shown for above value of $\beta$,

$$
\|U\|=\sqrt{\sum_{r \in \Omega} u_{r}(r)}=0.6927241623 \mathrm{~m} / \mathrm{s}
$$

The results and trends in Figure 2 [17] agree reasonably well with the present results in a small neighborhood of $t=0$. The continuity equation Equation (13) was also used to show that $A$ above is an acceptable solution for $\phi_{l}$ and $u_{r}$. 


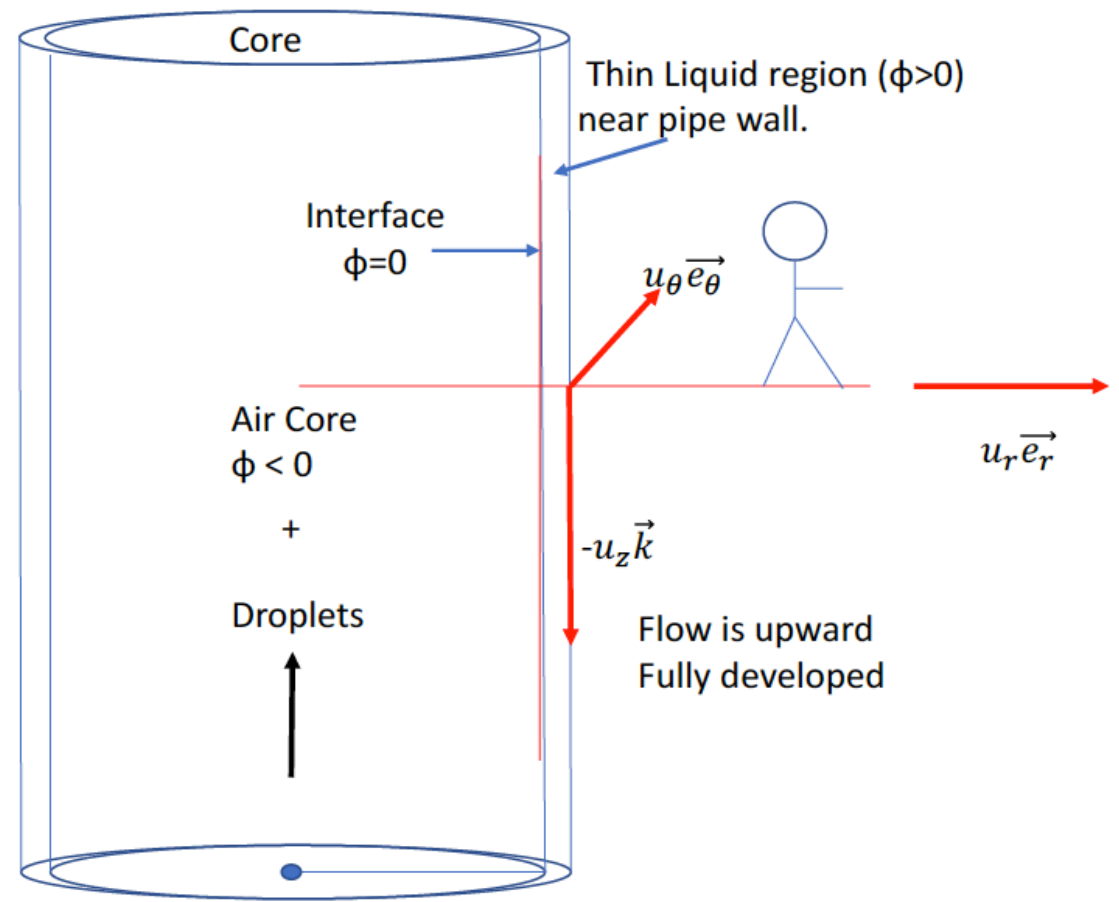

Figure 1. Two Phase Upward Co-current Annular Flow Geometry.

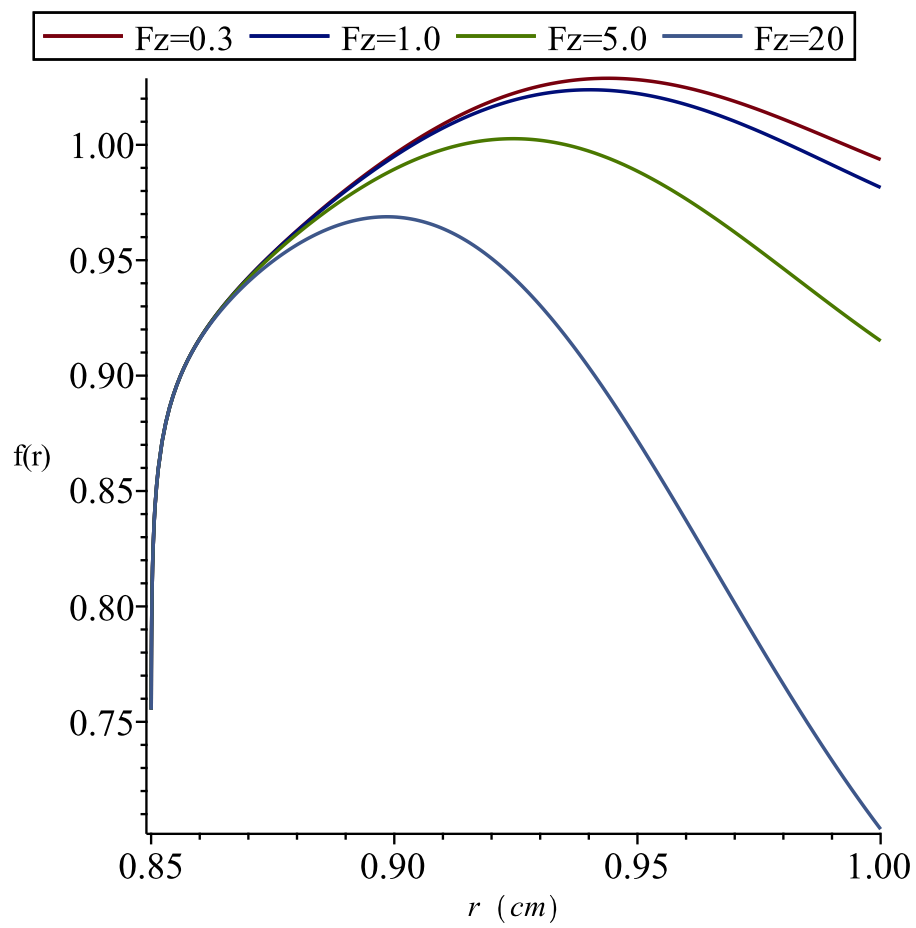

Figure 2. $f$ versus $r$ for $\alpha=-1000$ in level set function $\phi, R_{i}=0.85 \mathrm{~cm}$ is the radial value at the gas/liquid interface. 


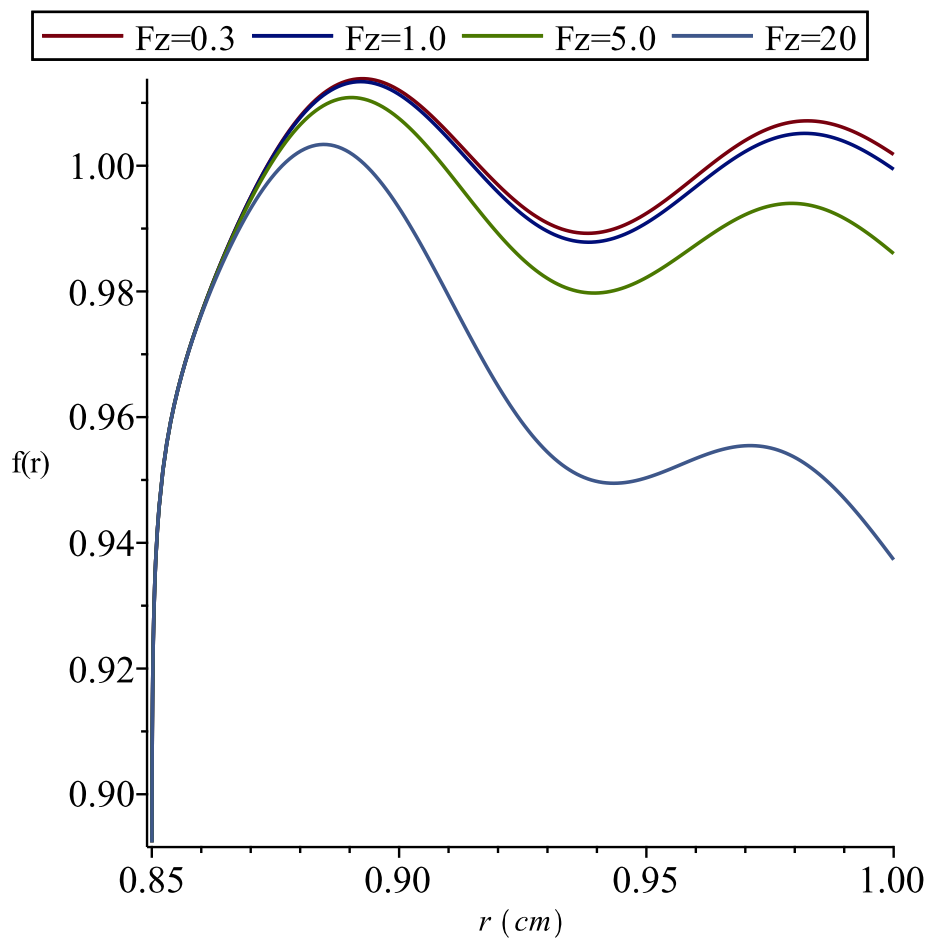

Figure 3. $f$ versus $r$ for $\alpha=-5000$ in level set function $\phi, R_{i}=0.85 \mathrm{~cm}$ is the radial value at the gas/liquid interface.

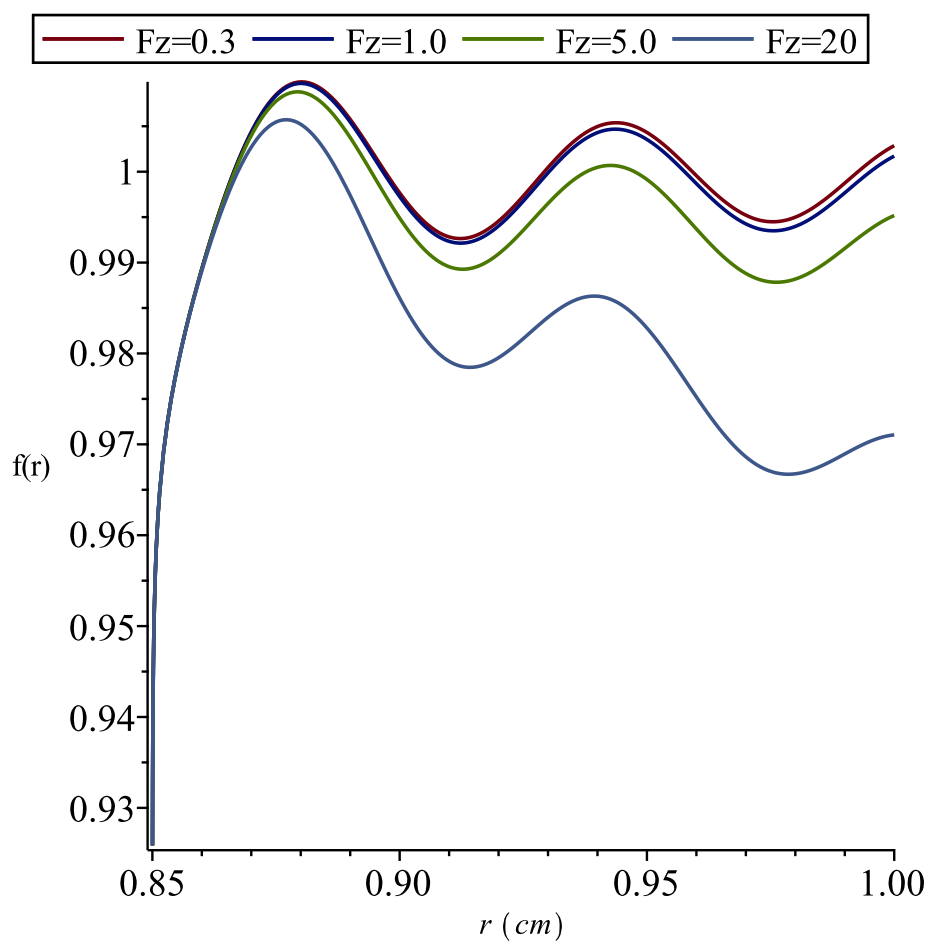

Figure 4. $f$ versus $r$ for $\alpha=-10,000$ in level set function $\phi, R_{i}=0.85 \mathrm{~cm}$ is the radial value at the gas/liquid interface. 


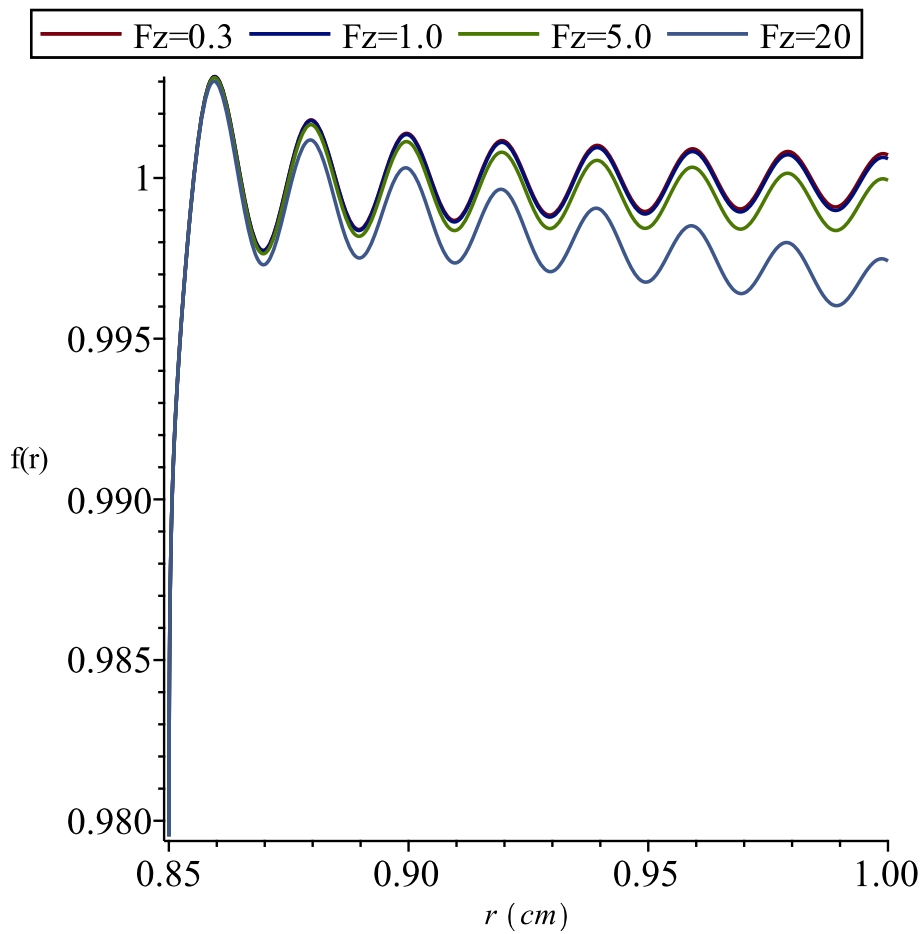

Figure 5. $f$ versus $r$ for $\alpha=-100,000$ in level set function $\phi, R_{i}=0.85 \mathrm{~cm}$ is the radial value at the gas/liquid interface.

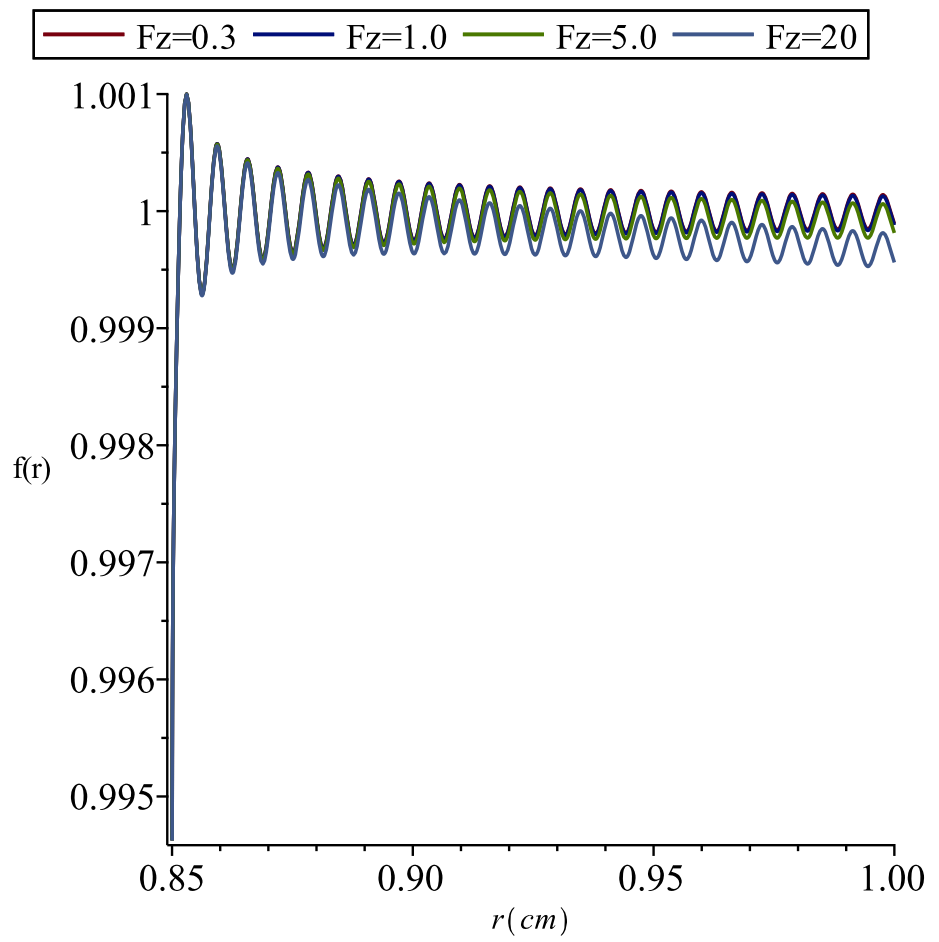

Figure 6. $f$ versus $r$ for $\alpha=-1,000,000$ in level set function $\phi, R_{i}=0.85 \mathrm{~cm}$ is the radial value at the gas/liquid interface. 


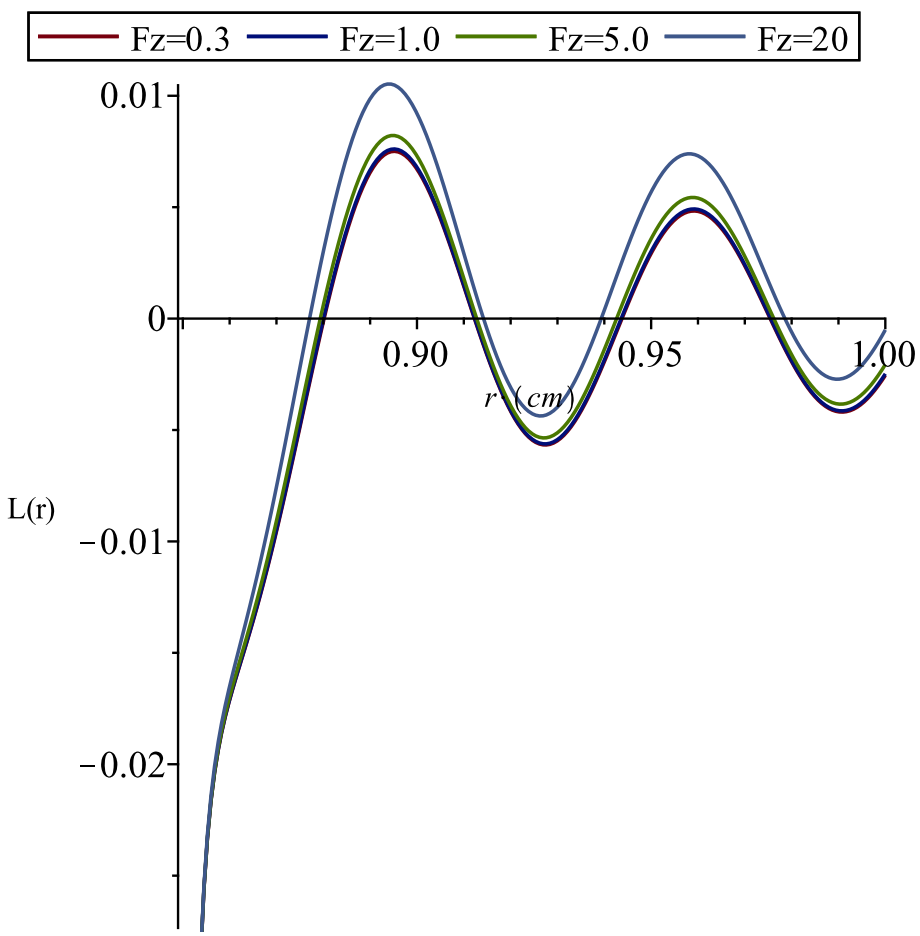

Figure 7. $L(r)$ versus $r$, for $\alpha=-10,000$ corresponding to Figure 4 at different values of $F z$.

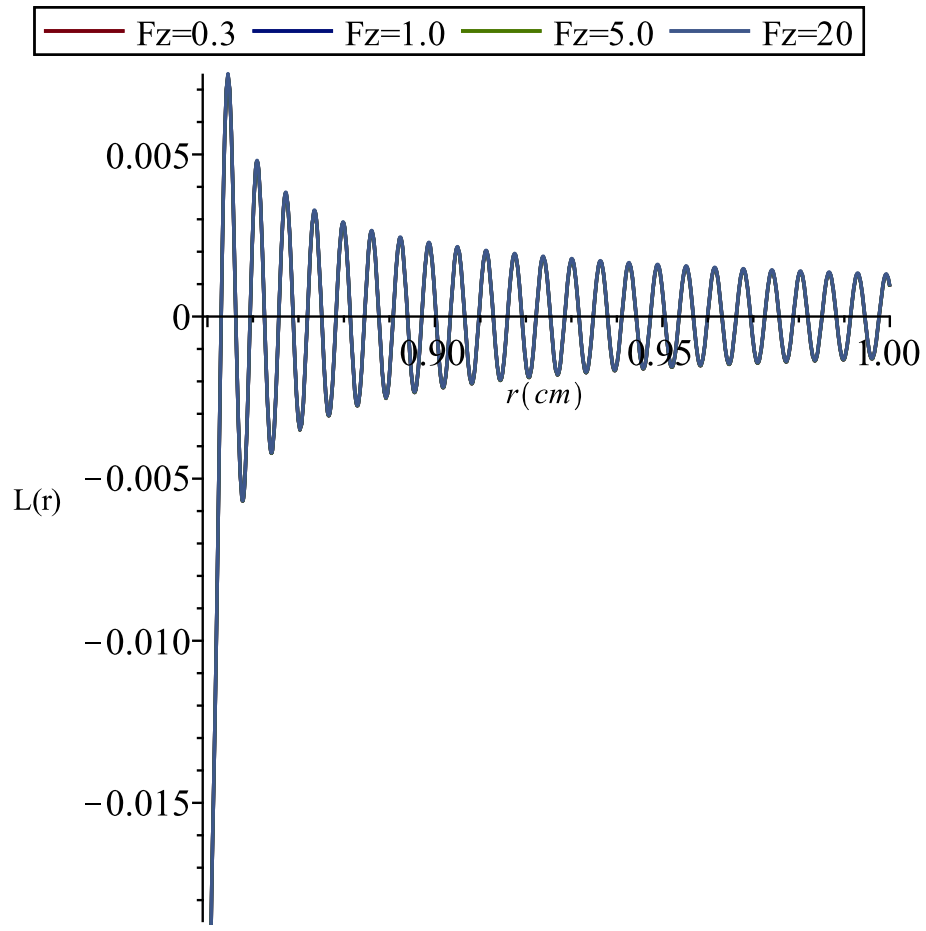

Figure 8. $L(r)$ versus $r$, for $\alpha=-1,000,000$ corresponding to Figure 6 at different values of $F z$.

\section{Conclusions}

It is worth noting that the interfacial oscillations occurring as extremes of two problems one for curvature coupled to composite velocity and the other for curvature alone presents the daunting problem of solving the full equation of Equation (25) without the assumption of small inclination angle. There is a plethora of results for computational multiphase flow using level set methods. The advantage 
of the present work lies in that analytical results are possible for the two extreme cases presented. It is conjectured at this point that the combination or full Equation (25) without the small inclination angle, i.e., approaching a horizontal tube configuration of flow, is non separable due to the inherent complexity of Equations (4) and (25) combined. We can expect that there be a very complex relationship between azimuthal and radial components of $\overline{\vec{L}}$. Work on the complete problem for this complex relationship is in progress for future studies.

Funding: This research received no external funding.

Acknowledgments: I would like to thank Zhen He ( Zhe265@uwo.ca) for some literature search support.

Conflicts of Interest: The author declares no conflict of interest.

\section{Appendix A}

Based on Equation (18) it is here shown that $\frac{\mu}{\rho}$ can be expressed as a partial fraction decomposition as follows:

$$
\begin{gathered}
\vec{L}=\frac{1}{\phi} \frac{\mu}{\rho} \overrightarrow{\vec{L}} \\
\vec{L}=\frac{\vec{L}}{\phi}\left(\frac{-\mu_{2}+\mu_{1}}{-\rho_{2}+\rho_{1}}+\frac{\mu_{1} \rho_{2}-\mu_{2} \rho_{1}}{\left(-\rho_{2}+\rho_{1}\right)\left(\rho_{1} \phi-\rho_{2} \phi-\rho_{1}\right)}\right)
\end{gathered}
$$

where $\mu=\mu_{1}+\mu_{D} \phi=\mu_{1}+\left(\mu_{2}-\mu_{1}\right) \phi$ and $\rho=\rho_{1}+\rho_{D} \phi=\rho_{1}+\left(\rho_{2}-\rho_{1}\right) \phi$. In Equation (A2) the partial fraction decomposition was found for the kinematic viscosity. For $\rho_{1}$ very small in magnitude relative to $\rho_{2}$ and $\mu_{1}$ very small in magnitude relative to $\mu_{2}$ we have

$$
\vec{L}=-\frac{\mu_{2}}{\rho_{2} \phi} \overline{\vec{L}}
$$

\section{References}

1. Adanhounme, V.; Adomoub, A.; Codo, F.P. Analytical solutions for Navier-Stokes equations in the cylindrical coordinates. Bull. Soc. Math. Serv. Stand. 2012, 2, 16-23. [CrossRef]

2. Pereira, L.M.; Guerrero, J.S.P.; Cotta, R.M. Integral transformation of the Navier-Stokes equations in cylindrical geometry. Comput. Mech. 1998, 21, 60-70. [CrossRef]

3. Taylor, C.; Hood, P. A numerical solution of the Navier-Stokes equations using the finite element technique. Comput. Fluids 1973, 1, 73-100. [CrossRef]

4. Chorin, A.J. The numerical solution of the Navier-Stokes equations for an incompressible fluid. Bull. Am. Math. Soc. 1967, 73, 928-931. [CrossRef]

5. Osher, S.; Sethian, J. Fronts propagating with curvature-dependent speed: Algorithms based on Hamilton-Jacobi formulations. J. Comp. Phys. 1988, 79, 12-49. [CrossRef]

6. Sethian, J. Level Set Methods and Fast Marching Methods; Cambridge University Press: Cambridge, UK, 1999.

7. Duflot, M. A study of the representation of cracks with level sets. Int. J. Numer. Methods Eng. 2007, 70, 1261-1302. [CrossRef]

8. Caselles, V.; Catte, F.; Coll, T.; Dibos, F. A geometric model for active contours in image processing. Numer. Math. 1993, 66, 1-31. [CrossRef]

9. Li, C.; Huang, R.; Ding, Z.; Gatenby, J.C.; Metaxas, D.N. A Level Set Method for Image Segmentation in the Presence of Intensity Inhomogeneities with Application to MRI. IEEE Trans. Image Process. 2011, 20, 2007-2016. [PubMed]

10. Mitchell, I.; Tomlin, C.J. Level Set Methods for Computation in Hybrid Systems, International Workshop on Hybrid Systems: Computation and Control. In Proceedings of the International Workshop on Hybrid Systems: Computation and Control, Pittsburgh, PA, USA, 23-25 March 2000; pp. 310-323.

11. Maitre, E.; Milcent, T.; Cottet, G.-H.; Raoult, A.; Usson, Y. Applications of level set methods in computational biophysics. Math. Comput. Model. 2009, 49, 2161-2169. [CrossRef] 
12. Sussman, M.; Smereka, P.; Osher, S. A level set approach for computing solutions to incompressible two-phase flow. J. Comput. Phys. 1994, 114, 146-159. [CrossRef]

13. Olsson, E.; Kreiss, G. A conservative level set method for two phase flow. J. Comput. Phys. 2005, 210, $225-246$. [CrossRef]

14. Ayati, A.A.; Farias, P.S.C.; Azevedo, L.F.A.; de Paula, I.B. Characterization of linear interfacial waves in a turbulent gas-liquid pipe flow. Phys. Fluids 2017, 29, 062106. [CrossRef]

15. Osher, S.; Fedkiw, R. Level Set Methods and Dynamic Implicit Surfaces; Springer: Berlin, Germany, 2003.

16. Yaji, K.; Otomori, M.; Yamada, T.; Izui, K.; Nishiwaki, S.; Pironneau, O. Shape and topology optimization based on the convected level set method. Struct. Multidiscip. Optim. 2016, 54, 659-672. [CrossRef]

17. Xie, F.; Zheng, X.; Triantafyllou, M.S.; Constantinides, Y.; Zheng, Y.; Karniadakis, G.E. Direct numerical simulations of two-phase flow in an inclined pipe. J. Fluid Mech. 2017, 825, 189-207. [CrossRef]

(C) 2018 by the authors. Licensee MDPI, Basel, Switzerland. This article is an open access article distributed under the terms and conditions of the Creative Commons Attribution (CC BY) license (http:// creativecommons.org/licenses/by/4.0/). 